For developing the Strategy, workshops were organized to bring stakeholders across HSE to review existing systems for conducting population surveys, collecting exposure intelligence and occupational health surveillance, which have contributed to forming a long-term vision of fit-for-purpose measurement systems.

We will present the development of the Strategy and the plans to implement it with the H\&W program, which requires close collaborations between epidemiologists and social researchers, policy makers, and other multidisciplinary regulatory specialists. The lessons learnt will help HSE towards building the right evidence base for monitoring and evaluation of a range of national level intervention programs for work-related ill health prevention.

(C) British Crown copyright (2019)

\section{C.3 EFFECTIVENESS OF A MULTI-FACETED INTERVENTION TO PREVENT MUSCULOSKELETAL PAIN IN NURSES AND AIDES: RESULTS OF A CLUSTER-RANDOMIZED CONTROLLED TRIAL}

1,2,3 Mercè Soler Font* 1,2,3 José Maria Ramada Rodilla ${ }^{4}$ Sander van Zon ${ }^{4}$ Josué Almansa Ortega, ${ }^{4}$ Ute Bültmann, ${ }^{1,2,3}$ Consol Serra Pujadas. ${ }^{1}$ Center for Research in Occupational Health, Department of Experimental and Health Sciences University Pompeu Fabra, Barcelona, Spain; ${ }^{2}$ CIBER of Epidemiology and Public Health, Spain, Barcelona, Spain; ${ }^{3}$ MIM (Hospital del Mar Medical Research Institute), Barcelona, Spain; ${ }^{4}$ Department of Health Sciences, Community and Occupational Medicine, University Medical Center Groningen, University of Groningen, Groningen, Groningen, The Netherlands

\subsection{6/OEM-2019-EPI.174}

Background Health workers are at high risk of developing musculoskeletal pain (MSP). This study aimed to evaluate a multi-faceted intervention in two public tertiary hospitals, encompassing three levels of prevention and health promotion to prevent and manage MSP.

Methods A two-armed cluster randomized controlled trial, with a late intervention control group was performed. Clusters were independent hospital units, and participants were the nursing staff. The intervention comprised three components: participatory ergonomics, case management, and health promotion. The control group received usual occupational health care. The intervention lasted one year and data were collected at baseline, 6 and 12 months follow-up. Primary outcomes were self-perceived MSP and its associated sickness absence. The process evaluation included recruitment, context, reach, dose administered and received, fidelity, satisfaction, implementation strategy, and discussion groups of experts and participants.

Results Eight clusters, including 445 participants, were randomized. In the intervention group a $20 \%$ statistically significant reduction of neck, shoulders and upper back pain compared to the control group $(\mathrm{OR}=0.37 ; 95 \% \quad \mathrm{CI}=0.14-0.96)$ was observed at 12 months follow-up. We found no significant differences in incidence and duration of sickness absence. Organizational culture (secondary outcome) improved significantly in the intervention group in the domains of 'formal safety audits', 'availability of information for safety at work', and 'involvement of workers in decisions that affect their safety and health' compared to the control group. The intervention was implemented with $96.6 \%$ fidelity, and participants' adherence was $75.5 \%$. Participant's satisfaction was 9.1/10.

Conclusions This intervention showed effectiveness to reduce MSP and improve organizational culture, through to an integral management of MSP. Although our results are modest, strategies should focus on multi-faceted interventions, and occupational health services might be excellent opportunity for.

\section{O7C.4 USE OF CONTROL MEASURES AMONG CONSTRUCTION WORKERS WHEN PERFORMING DUSTY WORK}

Trang Khieu*, Jacob Daubé*. WorSafe New Zealand, Wellington, New Zealand

\subsection{6/OEM-2019-EPI.175}

Respirable dust, including respirable crystalline silica (RCS), is a common risk to health for construction workers. New Zealand and international literature has shown that construction workers are likely exposed to high levels of dust in the performance of their work. However, very little is known about the prevalence of controls used to minimise the dust exposure. An on-site survey of 250 construction workers was undertaken in Hamilton, New Zealand. Respondents were asked how frequently they completed any of six common high-risk activities for dust exposure: [1] cutting or drilling concrete, [2] grinding or polishing concrete, [3] jackhammering, [4] crushing concrete, [5] cutting or sanding wood, [6] cutting or sanding plasterboard or fibre cement board. For each activity completed more than once a month, workers were asked about the controls they used to minimise exposure to dust and their consideration of the risks to their health. Low levels of controls were reported across the high-risk activities. The majority of workers did not usually use water suppression when jackhammering $(79.6 \%)$ or crushing concrete $(56.3 \%)$. Nearly two thirds $(62.8 \%)$ of workers did not use dry dust extraction when cutting or sanding wood, and over a quarter of respondents used neither water suppression nor dry dust extraction when cutting/drilling concrete (30.1\%) or grinding/ polishing concrete $(25.0 \%)$. Half of the workers $(50 \%)$ often or always wore respirators when performing dusty work but fewer than half of ever-wearers were fit-tested or clean-shaven. Younger workers ( $<25$ years) were significantly less likely to consider risks to their health or wear a respirator compared to older workers $(\geq 25)$. This study provides an insight into the use of dust controls in the construction industry in New Zealand. Future work should investigate how to encourage or enable uptake of stronger dust controls.

\section{C.5 ASSESSING THE IMPACT OF INTERVENTION ON FUTURE LUNG CANCER BURDEN AMONG CONSTRUCTION WORKERS}

${ }^{1}$ Chaojie Song*, ${ }^{1}$ Kate Jardine, ${ }^{1,2}$ Victoria Arrandale, ${ }^{3,4}$ Young Jung, ${ }^{3,5} \mathrm{Amir}$ Mofidi, ${ }^{3}$ Emile Tompa, ${ }^{6}$ Thomas Tenkate, ${ }^{7,8}$ Hugh Davies, ${ }^{1,2,8}$ Paul A Demers. ${ }^{1}$ Occupational Cancer Research Centre, Cancer Care Ontario, Toronto, Canada; ${ }^{2}$ Dalla Lana School of Public Health, University of Toronto, Toronto, Canada; ${ }^{3}$ Institute for Work and Health, Toronto, Canada; ${ }^{4}$ McMaster University, Toronto, Canada; ${ }^{5}$ Department of Occupational Health Engineering, Tarbiat Modares University, Tehran, Iran; ${ }^{6}$ School of Occupational and Public Health, Ryerson University, Toronto, Canada; ${ }^{7}$ CAREX Canada, Burnaby, Canada; ${ }^{8}$ School of Population and Public Health, University of British Columbia, Vancouver, Canada

\subsection{6/OEM-2019-EPI.176}

Background and objectives Construction workers are exposed to several carcinogens at work. Implementing intervention methods may reduce workers' exposure, which should subsequently reduce the number of cancer cases attributable to the 